\title{
Nicotine Risk Misperception Among US Physicians
}

J Gen Intern Med 36(12):3888-90

DOI: $10.1007 / \mathrm{s} 11606-020-06172-8$

(C) Society of General Internal Medicine 2020

\section{INTRODUCTION}

Nicotine is responsible for the highly addictive nature of tobacco products, but most tobacco-caused disease is not directly caused by nicotine, but rather by other chemicals present in tobacco or tobacco smoke. ${ }^{1}$ The strongest evidence for direct causality for nicotine is for birth defects (neurodevelopment), with only limited evidence supporting causal links to cancer and cardiovascular disease, and scarce data for COPD. Despite this, many misperceive nicotine as responsible for smoking-related health risks, like cancer. ${ }^{2,3}$ These misperceptions are not unique to the general population; in one study, $60 \%$ of nurses incorrectly perceived nicotine as carcinogenic. ${ }^{4}$ This study explores physician perceptions of nicotine risk in a national survey.

\section{METHODS}

This analysis represents a national mail survey exploring physicians' knowledge and communication about tobacco use, collected between September 2018 and February 2019. The survey targeted six specialties: family medicine, internal medicine, $\mathrm{OB} / \mathrm{GYN}$, cardiology, pulmonary/critical care, and hematology/oncology. The sample was drawn from the AMA Physician Masterfile, and 500 physicians from each specialty were randomly selected. Overall, 1058 physicians participated and 496 physicians were ineligible (e.g., not seeing outpatients), yielding an AAPOR response rate of $51.8 \%$. The survey domains included tobacco treatment practices, harm reduction beliefs, and tobacco/e-cigarette knowledge. Physicians also rated (on a 4-point scale) if they agreed or disagreed that "nicotine directly contributes to": the development of birth defects; cardiovascular disease; cancer; and COPD. We estimated prevalence and adjusted prevalence ratios (APRs) for strongly agreeing that nicotine causes each outcome as a function of physician gender, age, and specialty using multivariable log-binomial regression models, controlling for race/ethnicity.

Received August 3, 2020

Accepted August 17, 2020

Published online September 1, 2020

\section{RESULTS}

Participant characteristics are summarized in Table 1. Overall, the majority of physicians "strongly agreed" that nicotine directly contributes to the development of cardiovascular disease (83.2\%), COPD (80.9\%), and cancer (80.5\%). Comparatively fewer "strongly agreed" that nicotine directly contributes to the development of birth defects (32.9\%) and 30.2\% did not answer this question - a potential indicator of "do not know."

Table 1 Sample Characteristics and Beliefs About Nicotine by Specialty, $N=1020$

\begin{tabular}{ll}
\hline \hline & $\boldsymbol{n}^{\mathrm{a}}(\boldsymbol{\%})$ \\
\hline Gender & \\
Female & $581(57.0)$ \\
Male & $439(43.0)$ \\
Race/ethnicity & $664(65.1)$ \\
White, non-Hispanic & $48(4.71)$ \\
Black, non-Hispanic & $40(3.92)$ \\
Hispanic & $213(20.9)$ \\
Asian/PI, non-Hispanic & $55(5.4)$ \\
Other/unknown, non-Hispanic & \\
Age & $51.5 \pm 10.6$ \\
Mean \pm SD & \\
Specialty & $144(14.1)$ \\
Cardiology & $202(19.8)$ \\
Family medicine & $164(16.1)$ \\
Internal medicine & $201(19.7)$ \\
OB/GYN & $136(13.3)$ \\
Oncology & $173(17.0)$ \\
Pulmonary & \\
Nicotine causes birth defects & $332(32.6)$ \\
Strongly agree & $223(21.9)$ \\
Somewhat agree & $101(9.9)$ \\
Somewhat disagree & $56(5.5)$ \\
Strongly disagree & $308(30.2)$ \\
Missing & \\
Nicotine causes cardiovascular disease & $850(83.3)$ \\
Strongly agree & $86(8.4)$ \\
Somewhat agree & $40(3.9)$ \\
Somewhat disagree & $40(3.9)$ \\
Strongly disagree & $4(0.4)$ \\
Missing & \\
Nicotine causes cancer & $821(80.5)$ \\
Strongly agree & $64(6.3)$ \\
Somewhat agree & $67(6.6)$ \\
Somewhat disagree & $65(6.4)$ \\
Strongly disagree & $3(0.3)$ \\
Missing & \\
Nicotine causes COPD & $825(80.9)$ \\
Strongly agree & $53(5.2)$ \\
Somewhat agree & $67(6.6)$ \\
Somewhat disagree & $71(7.0)$ \\
Strongly disagree & $4(0.4)$ \\
Missing & \\
\hline \\
We exclucd
\end{tabular}

${ }^{a}$ We excluded 38 participants with missing responses for one or more analysis variables

${ }^{b}$ Age was imputed for 21 participants as the median age of other respondents of the same specialty and graduation year $C O P D$, chronic obstructive pulmonary disease 
Table 2 Prevalence and Adjusted Associations with Belief That Nicotine Directly Contributes to Development of Birth Defects, CVD, Cancer, and COPD $(N=\mathbf{1 0 2 0})$

\begin{tabular}{|c|c|c|c|c|c|c|c|c|}
\hline & \multicolumn{2}{|c|}{ Birth defects } & \multicolumn{2}{|l|}{ CVD } & \multicolumn{2}{|l|}{ Cancer } & \multicolumn{2}{|l|}{ COPD } \\
\hline \multicolumn{9}{|l|}{ Gender } \\
\hline Male & $27.6 \%$ & Referent & $85.9 \%$ & Referent & $82.9 \%$ & Referent & $84.1 \%$ & Referent \\
\hline \multicolumn{9}{|l|}{ Age } \\
\hline 5 years & - & $0.91(0.88,0.96)$ & - & $0.99(0.98,1.00)$ & - & $0.99(0.98,1.00)$ & - & $0.99(0.98,1.01)$ \\
\hline Internal medicine & $39.6 \%$ & $1.86(1.35,2.57)$ & $81.7 \%$ & $0.94(0.85,1.04)$ & $82.9 \%$ & $1.08(0.96,1.21)$ & $83.5 \%$ & $1.22(1.08,1.38)$ \\
\hline $\mathrm{OB} / \mathrm{GYN}$ & $21.4 \%$ & Referent & $87.6 \%$ & $1.00(0.92,1.09)$ & $83.1 \%$ & $1.08(0.97,1.21)$ & $85.1 \%$ & $1.25(1.11,1.40)$ \\
\hline Oncology & $30.9 \%$ & $1.34(0.93,1.93)$ & $80.2 \%$ & $0.93(0.83,1.03)$ & $77.2 \%$ & Referent & $76.5 \%$ & $1.12(0.98,1.29)$ \\
\hline Pulmonary & $32.4 \%$ & $1.42(1.01,1.99)$ & $73.4 \%$ & $0.84(0.75,0.94)$ & $68.2 \%$ & $0.89(0.78,1.02)$ & $68.2 \%$ & Referent \\
\hline
\end{tabular}

Prevalence ratios are adjusted for all variables in table and racelethnicity (NH Black, Hispanic, NH Asian, NH other/unknown vs. NH White). We excluded 38 participants with missing responses for one or more analysis variables

$C V D$, cardiovascular disease; COPD, chronic obstructive pulmonary disease; APR, adjusted prevalence ratio; CI, confidence interval

Misperceptions regarding nicotine's role differed by physician characteristics (Table 2). Females were more likely than males to correctly perceive nicotine risks for birth defects (APR 1.28, 95\% CI 1.07-1.54). Younger physicians were also more likely to correctly perceive the impact on birth defects. Pulmonologists were less likely than most other specialties to misperceive nicotine as a direct contributor to COPD. Additionally, family physicians were more likely than oncologists to misperceive nicotine as a carcinogen. Paradoxically, OB/ GYNs misidentified risk related to birth defects more than other specialties.

\section{DISCUSSION}

To our knowledge, this is the first study to document widespread nicotine misperceptions among physicians in the USA. While it is possible that some physicians may have misunderstood the question (e.g., considered harm caused by tobacco, rather than nicotine), results are consistent with other studies finding notable nicotine misperceptions. ${ }^{2-4}$ Correcting misperceptions should be a priority given that in 2017 the FDA proposed a nicotine-centered framework that includes reducing nicotine content in cigarettes to non-addictive levels while encouraging safer forms of nicotine use for either harm reduction (e.g., smokeless tobacco) or cessation (pharmacologic NRT). ${ }^{5}$ Short communication interventions can effectively correct such nicotine misperceptions. ${ }^{6}$ It is vital that physicians understand the actual risk of nicotine given that they are critical in the prescribing and recommendation of FDAapproved NRT products. Moreover, so they can accurately communicate risk in an evolving tobacco marketplace which may include low-nicotine cigarettes, which are not safer than traditional cigarettes. To best serve patients, physicians need to be better informed that the primary risk of nicotine in tobacco products is due to addiction/dependence, while other carcinogens and chemicals, particularly those produced by combustion, serve as the primary source of risk for tobaccocaused diseases. ${ }^{1}$

Access to Data. CDD, MBTM, and MBS had full access to all the data in the study and take responsibility for the integrity of the data and the accuracy of the data analysis. All authors contributed to preparation of the manuscript.

Michael B. Steinberg, MD, $M P H^{1}$

Michelle T. Bover Manderski, PhD, MPH ${ }^{2}$

Olivia A. Wackowski, PhD, $M P H^{2}$

Binu Singh, $M P H^{2}$

Andrew A. Strasser, $P h D^{3}$

Cristine D. Delnevo, $\mathrm{PhD}, \mathrm{MPH}^{2}$

${ }^{1}$ Department of Medicine, Robert Wood Johnson Medical School, Rutgers University,

New Brunswick, NJ, USA

${ }^{2}$ Rutgers Center for Tobacco Studies, Rutgers University,

New Brunswick, NJ, USA

${ }^{3}$ Department of Psychiatry, Perelman School of Medicine, University of Pennsylvania,

Philadelphia, PA, USA

Corresponding Author: Michael B. Steinberg, MD, MPH; Department of Medicine, Robert Wood Johnson Medical School, Rutgers University, New Brunswick, NJ, USA (e-mail: michael.steinberg@rutgers.edu).

Funding This work was supported by grant 1R01CA190444-01A1 from the National Cancer Institute (NCI). Additionally, $C D D, O A W$, and AAS were supported by Tobacco Centers of Regulatory Science award U54CA229973 from the National Cancer Institute (NCI) and Food and Drug Administration (FDA).

\section{Compliance with Ethical Standards:}

Conflict of Interest: The authors declare that they do not have a conflict of interest. 
Disclaimer: The content of this manuscript is solely the responsibility of the authors and does not necessarily represent the official views of the National Institutes of Health or the US Food and Drug Administration. The funder had no role in the design and conduct of the study; collection, management, analysis, and interpretation of the data; preparation, review, or approval of the manuscript; or decision to submit the manuscript for publication.

\section{REFERENCES}

1. National Center for Chronic Disease Prevention and Health Promotion (US) Office on Smoking and Health. The Health Consequences of Smoking-50 Years of Progress: A Report of the Surgeon General. Atlanta (GA): Centers for Disease Control and Prevention (US); 2014.

2. Cummings KM, Hyland A, Giovino GA, Hastrup JL, Bauer JE, Bansa MA. Are smokers adequately informed about the health risks of smoking and medicinal nicotine?. Nicotine Tob Res. 2004;6 Suppl 3:S333-S340. https://doi.org/10.1080/14622200412331320734.
3. O'Brien EK, Nguyen AB, Persoskie A, Hoffman AC. U.S. adults' addiction and harm beliefs about nicotine and low nicotine cigarettes. Prev Med. 2017;96:94-100. https://doi.org/10.1016/j.ypmed.2016.12. 048.

4. Borrelli B, Novak SP. Nurses' knowledge about the risk of light cigarettes and other tobacco "harm reduction" strategies. Nicotine Tob Res. 2007;9(6):653-661. https://doi.org/10.1080/14622200701365202.

5. Gottlieb S, Zeller M. A Nicotine-Focused Framework for Public Health. N Engl J Med. 2017;377(12):1111-1114. https://doi.org/10.1056/ NEJMp1707409.

6. Villanti AC, West JC, Mays D, Donny EC, Cappella JN, Strasser AA. Impact of Brief Nicotine Messaging on Nicotine-Related Beliefs in a U.S. Sample. Am J Prev Med. 2019;57(4):e135-e142. https://doi.org/10.1016/ j.amepre.2019.05.015

Publisher's Note: Springer Nature remains neutral with regard to jurisdictional claims in published maps and institutional affiliations. 